\title{
Postoperative pulmonary artery stenosis in lung transplantation recipients: review of case series
}

\author{
Seung Hyun Yong ${ }^{1}$, Song Yee Kim ${ }^{1}$, Jin Gu Lee ${ }^{2}$, Hyo Chae Baek², Moo Suk Park ${ }^{1}$ \\ ${ }^{1}$ Division of Pulmonology, Department of Internal Medicine, Yonsei University College of Medicine, Seoul, Korea \\ ${ }^{2}$ Department of Thoracic and Cardiovascular Surgery, Yonsei University College of Medicine, Seoul, Korea
}

Background: Postoperative pulmonary artery stenosis (PAS) is rare complication that can appear lung transplant recipients due to inappropriate donor to recipient lung size matching. In this study, we retrospectively reviewed patients who were diagnosed PAS after lung transplantation.

Methods: Medical records were retrospectively reviewed in the group of lung transplant recipients from 2012 to 2019 in Severance Hospital, Korea.

Results: Among 267 recipients who underwent lung transplantation from October 2012 to December 2019, 12 patients were diagnosed PAS and treated accordingly. Four patients received surgical treatment. Six patients were successfully received interventional therapy: pulmonary angioplasty or pulmonary artery (PA) stent insertion. Two patients were under observation due to benign progress. Mortality were observed in three patients, and one death was related to PAS. Three patients had either left or right donor-to-recipient PA mismatch and four patients had donor lung size mismatch. After excluding the mortality and observant cases, eight patients had relief of symptoms and five patients showed increased outcome in before-and-after perfusion scan. Most common initial manifestation was progressive dyspnea and chest discomfort (seven cases), while median time of diagnosis-to-treatment was 36 days. Seven patients were diagnosed PAS in 2 weeks from day of lung transplantation. In this group, most common clue for diagnosis was acute change of chest X-ray (increased haziness) and hypoxemia. On contrary, five patients who were diagnosed after 2 weeks tended to not show acute change in chest $\mathrm{X}$-ray, rather most common phenomenon was progressive dyspnea, chest discomfort, and prolonged pneumonia.

Conclusions: PAS after lung transplantation can be managed by either PA intervention or surgical approach depending on circumstance of patients. Mortality were most likely associated with underlying patient's condition such as septic shock. Progressive dyspnea, chest discomfort, hypoxemia, and left lung haziness in chest X-ray may indicate PAS. Early diagnosis-to-treatment is vital for favorable outcome.

Corresponding author: Moo Suk Park

E-mail:PMS70@yuhs.ac

(C) The Korean Society for Transplantation

This is an Open Access article distributed under the terms of the Creative Commons Attribution Non-Commercial License (http://creativecommons.org/licenses/by-nc/4.0/) which permits unrestricted non-commercial use, distribution, and reproduction in any medium, provided the original work is properly cited. 